\title{
OH EGOs: Hydroxyl masers in Extended Green Objects
}

\section{Daysi Quinatoa*†}

Quito Astronomical Observatory, National Polytechnic School, Av. Gran Colombia S/N, Interior del Parque La Alameda, 170136, Quito, Ecuador

E-mail: daysi.quinatoa@epn.edu.ec

\section{Ross Burns}

Mizusawa VLBI Observatory, National Astronomical Observatory of Japan, 2-21-1 Osawa, Mitaka, Tokyo 181-8588, Japan

Joint Institute for VLBI ERIC, Oude Hoogeveensedijk 4, 7991 PD Dwingeloo, The Netherlands. E-mail: ross.burns@nao.ac.jp

\section{Katharina Immer}

Joint Institute for VLBI ERIC, Oude Hoogeveensedijk 4, 7991 PD Dwingeloo, The Netherlands. E-mail: immerejive.eu

\section{Olga Bayandina}

Astro Space Center, Lebedev Physical Institute, Russian Academy of Sciences, 84/32

Profsoyuznaya st., Moscow, 117997, Russia.

E-mail: bayandinalasc.rssi.ru

\section{Mateusz Olech}

Centre for Astronomy, Faculty of Physics, Astronomy and Informatics, Nicolaus Copernicus University, Grudziadzka 5, PL-87-100 Torun, Poland.

E-mail: olecheastro.uni.torun.pl

Extended green objects (EGOs) are a class of high-mass protostars characterised by active outflows seen in Spitzer data. The launching mechanism of outflows in massive embedded objects remains one of the key puzzles of massive star formation theory as it is thought to reflect characteristics of the accretion process. A sample of EGOs exhibiting $\mathrm{OH}$ masers were identified by VLA observations, raising the possibility of probing the magnetic fields at the launching region. This contribution introduces new high-resolution full-Stokes EVN observations of $\mathrm{OH}$ masers in EGOs targeting the launching region of outflows in high-mass protostars.

14th European VLBI Network Symposium \& Users Meeting (EVN 2018)

8-11 October 2018

Granada, Spain

\footnotetext{
* Speaker.

$\dagger$ Daysi Quinatoa would like to acknowledge the support of the ASTRON/JIVE International Summer Student Programme. The Joint Institute for VLBI ERIC, is a European entity established by six countries and funded by ten agencies to support the use of the European VLBI Network.
} 


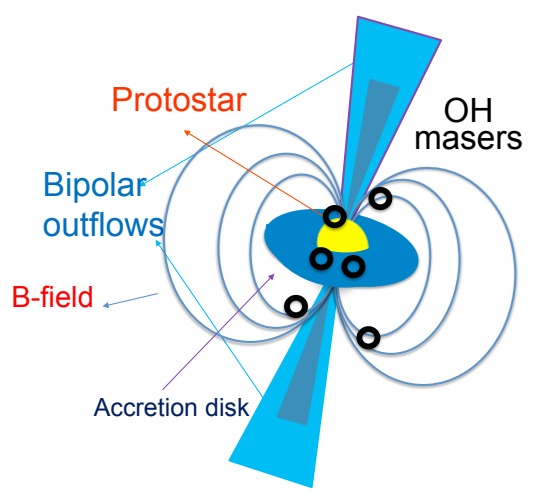

Figure 1: Schematic diagram of a prototypical embedded, accreting massive star. OH masers residing near key features involved in accretion and ejection may be used to probe the role of magnetic fields in the formation of massive stars.

\section{Introduction}

A complete understanding of massive star formation still eludes astronomers. While diskaided accretion in massive young stellar objects(MYSOs) is fast becoming accepted by the scientific community as a means of unifying high- and low-mass star formation some fundamental issues remain. Particularly, the nature of the accretion-ejection relation and the collimation of jets. Both of the aforementioned phenomena require magnetic fields as essential components in their theoretical framework.

The direction of the magnetic field can reveal phenomena such as toroidal jet fields (as in evolved stars[1]) - an essential proponent in magneto-centrifugal jets and angular momentum extraction. B-fields are also observed to thread disks (also implicated in disk- and X-wind launching) and magnetically regulate infall[2]. The onset of jet collimation is thought to happen shortly after emergence from the central region[3]. Furthermore, magnetic pressure must dominate gas dynamic pressure by 2-200 times for magnetic jet collimation[4]. These scenarios can be tested by polarimetry of the central regions of MYSOs.

At large distances from the star the toroidal magnetic field dominates over the poloidal field [5]. Such regions are best traced by water and Class I methanol masers which associate with shocks. The study of magnetic fields close to the accretion and jet-launching zone is better approached with masers which are pumped by FIR radiation close to the star and in the disk[6]. While OH masers are typically found on the peripheries of expanding (UC/)HII regions occasionally $\mathrm{OH}$ masers are found much closer to the central object. A variety of such objects was found in the sample of Bayandina et al. [7] who mapped $\mathrm{OH}$ emission in a sample of extended green objects (EGOs) [8], which are young, massive stars with bipolar outflows.

Hydroxyl $(\mathrm{OH})$ molecules respond to local magnetic fields by Zeeman splitting to a larger degree than other masing species and have well known Zeeman coefficients and thus are good magnetometers. Their large circular and linear polarisations reveal the magnetic field strengths and directions in the line of sight and sky-plane, giving the 3D B-field structure. High resolution observations of $\mathrm{OH}$ masers permit investigations of magnetic fields at $\mathrm{AU}$ scales - similar to the 
launching regions of MYSOs (a few tens of AU - e.g. [9]). In these proceedings, we present early results from a campaign investigating the magnetic field properties of EGOs via VLBI polarisation mapping observations of the $1665 \mathrm{MHz}$ maser line.

\section{Observations}

Based on the spectral results of a preliminay detection survey, G47.47+0.05 was selected as the initial target. The source is located on the northern border of the extended HII region, G45L, at a distance of $8.3 \mathrm{kpc}$ ([10], and references therein). Observations were carried out with the European VLBI Network (EVN) on 2017 November 3, at 1.6 GHz. The participating stations were Jodrell Bank, Westerbork, Effelsberg, Medicina, Onsala, Tianma, Torun, Svetloe, Badary, Zelenchukskaya, Hartebeesthoek and Irbene. The observations lasted 6 hrs, using one frequency channel of 16-MHz bandwidth and dual polarisation. Stations recorded data to disks, which were shipped to JIVE and correlated with the SFXC[11].

Data analysis, calibration and imaging were performed using the Astronomical Image Processing System(AIPS). Amplitude and delay calibration of the spectral line data was derived from scans of the continuum source J2202+4216 (BLLAC), and phase and rate fringe fitting was performed on a strong maser feature. Imaging the spectral data was carried out with CLEAN algorithm and maps of the left and right circular polarisation were produced independently.

\section{Discussion}

We present the first results from full-track observations of G45.47+0.05. The EVN observations revealed that the spectrum in both LCP and RCP polarisations has a double-peak shape, displaced from each other due to a Zeeman splitting[6]. It is clearly seen that the LCP and RCP spectra are not similar in shape so it does not correspond to a simple case of Zeeman splitting. Also, the spectrum shows that the LCP polarization is more intense than the RCP polarization.

The Zeeman effect gives rise to three lines: two $\sigma$ components of opposite elliptical polarization (Zeeman pairs) displaced in frequency proportional to the magnetic field strength along the line of sight, and a component $\pi$ which provides information about sky-plane magnetic field. In this project, we identify a Zeeman pair as two masers at the same coordinate position but some offset in each of the circular polarizations.

G45.47+0.05 exhibits five Zeeman pairs based on the EVN spectra and maser spot maps. The intensity of the magnetic field range from $-1.49 \mathrm{mG}$ to $4.77 \mathrm{mG}$, which indicates the presence of strong magnetic fields. This value is consistent with magnetic field strength reported by VLA survey[6]. Similarity between the VLA and EVN results suggests that either the field is dominated by small scale components that are unresolved by both instruments, or that the field properties are similar at extended and compact scales; they are not scale dependant.

The magnetic field direction(+ or - sign) is seen to invert even within small physical space - suggesting a turbulent field rather than a large-scale, uniform field. The change of sign in the magnetic field may represent differences seen across the opposite sides of the accretion disk, or contributions from the jet launching region. 
Future work will concentrate on the characterisation of the sky-plane magnetic field via linear polarisation estimates to provide the 3D direction and strength of B-fields. Also the current investigation will be extended to additional EGOs (G34.403+0.233 and G35.03+0.35). This study is the first step of the 'OH EGOs' project to determine the importance of B-fields in the formation of massive stars using Hydroxyl masers in EGOs.

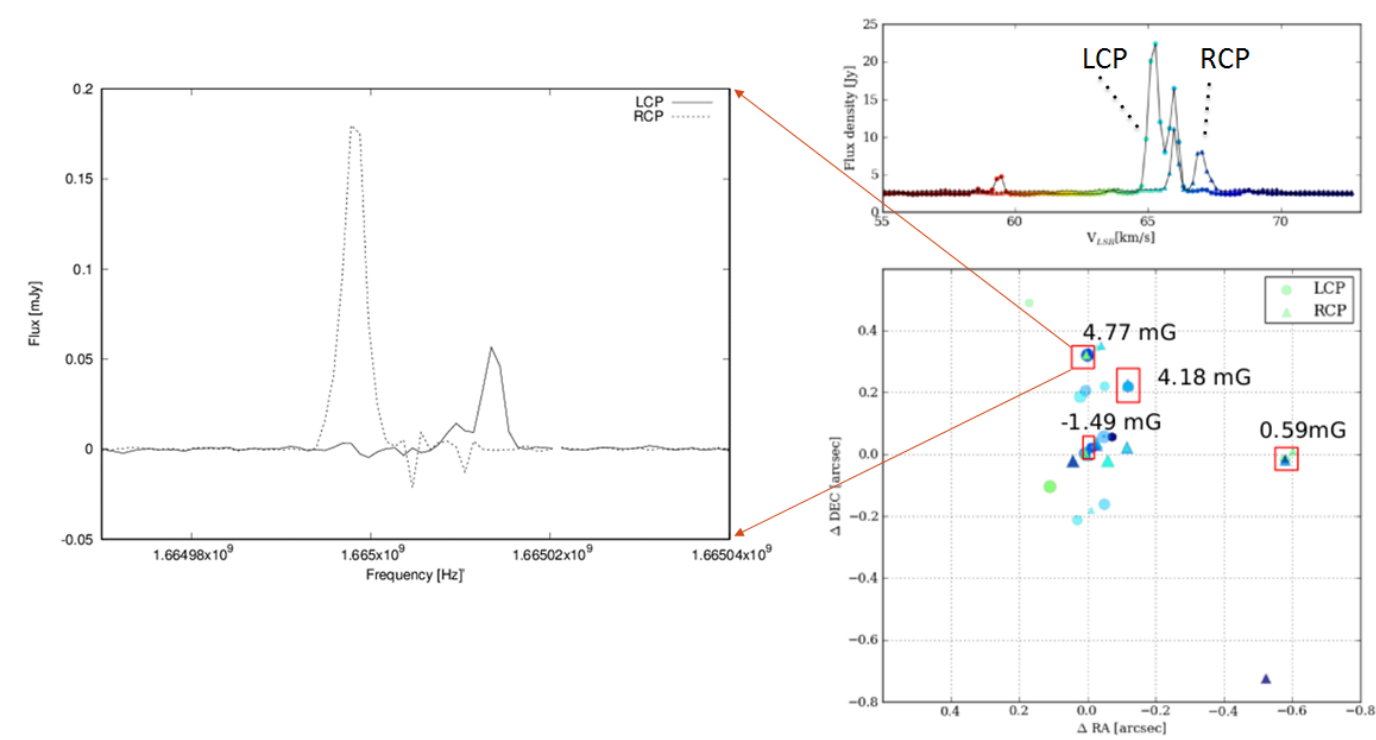

Figure 2: $\mathrm{OH}$ masers in G45.47+0.05, showing strong Zeeman splitting. The spectrum (AIPS task ISPEC) of one Zeeman pair is highlighted, indicating a B-field of $+4.7 \mathrm{mG}$ (left).

\section{References}

[1] Vlemmings, W. H. T., Diamond, P. J., van Langevelde, H. J. and Torrelles, J. M., The magnetic field in the star-forming region Cepheus A - from H2O maser polarization observations, A\&A 448 (2006) 597.

[2] W. H. T. Vlemmings, G. Surcis, K. J. E. Torstensson and H. J. van Langevelde, Magnetic field regulated infall on the disc around the massive protostar CepheusAHW2, 404 (2010) 134 [1002.2214].

[3] C. Carrasco-González, J. M. Torrelles, J. Cantó, S. Curiel, G. Surcis, W. H. T. Vlemmings et al., Observing the onset of outflow collimation in a massive protostar, Science 348 (2015) 114 [1507.05285].

[4] G. García-Segura, J. A. López and J. Franco, Magnetically Driven Winds from Post-Asymptotic Giant Branch Stars: Solutions for High-Speed Winds and Extreme Collimation, 618 (2005) 919 [astro-ph/0409595].

[5] L.-X. Li, Jet Collimation by Small-Scale Magnetic Fields, 564 (2002) 108 [astro-ph/ 0108469 ].

[6] O. Bayandina, P. Colom, S. Kurtz, G. Larionov, G. M. Rudnitskij, N. N. Shakhvorostova et al., Probing glimpse Extended Green Objects (EGOs) with hydroxyl masers, In prep. (2019) . 
[7] O. S. Bayandina, I. E. Val'tts and S. E. Kurtz, High resolution OH observations of Extended Green Objects, Astronomy Reports 59 (2015) 998.

[8] C. J. Cyganowski, B. A. Whitney, E. Holden, E. Braden, C. L. Brogan, E. Churchwell et al., A Catalog of Extended Green Objects in the GLIMPSE Survey: A New Sample of Massive Young Stellar Object Outflow Candidates, 136 (2008) 2391 [0810 . 0530].

[9] M. M. Wright, M. D. Gray and P. J. Diamond, The OH ground-state masers in W3(OH) - II. Polarization and multifrequency results, 350 (2004) 1272.

[10] M. J. Reid, K. M. Menten, A. Brunthaler, X. W. Zheng, T. M. Dame, Y. Xu et al., Trigonometric Parallaxes of High Mass Star Forming Regions: The Structure and Kinematics of the Milky Way, 783 (2014) 130 [1401.5377].

[11] A. Keimpema, M. M. Kettenis, S. V. Pogrebenko, R. M. Campbell, G. Cimó, D. A. Duev et al., The SFXC software correlator for very long baseline interferometry: algorithms and implementation, Experimental Astronomy 39 (2015) 259 [1502.00467]. 